\title{
Föderalismus und Regionalpolitik
}

Die Schweiz ist bekanntlich ein Bundesstaat, geprägt durch den Föderalismus. Die Regionalpolitik muß sich mit ihm auseinandersetzen. Was bedeutet der Föderalismus für die Regionalpolitik? Ist der Föderalismus «Gegenstand» oder "Voraussetzung» der Regionalpolitik? Am Beispiel der Schweiz läßt sich verdeutlichen, wie sich Föderalismus und Regionalpolitik zueinander verhalten.

\section{Die föderalistisch konzipierte Schweiz}

Der schweizerische Bundesstaat, auch Schweizerische Eidgenossenschaft genannt, gliedert sich - der Föderalismus strukturiert - in den Bund, in 26 Kantone und darüber hinaus in rund $3000 \mathrm{Gemeinden}$. Die Kantone behaupten von sich, sie seien souverän. Die Bundesverfassung unterstreicht dies, wenn auch mit Vorbehaten (Art. $3 \mathrm{BV}$ ). Die Gemeinden beanspruchen zusätzlich einen hohen Grad an Autonomie. Dessenungeachtet konzentriert sich die Aufgabenfülle in steigendem Maße beim Bund. Wichtige öffentliche Funktionen sind ihm anvertraut, so die Außen- und Sicherheitspolitik, aber auch große Teile der Verkehrs-, Umwelt-, Sozial- und Finanzpolitik. Für die Kantone stehen die Kultur-, Raumordnungs-, Gesundheits-, Straßenbau- und Steuerpolitik sowie das Polizeiwesen im Vordergrund wie auch der Vollzug der Bundesgesetzgebung, zumal der Bund - in wichtigen Bereichen - nicht über eine eigene Vollzugsverwaltung verfügt.

Die föderalistisch konzipierte Schweiz ist gleichzeitig Rechtsstaat und Demokratie; sie ist der Marktwirtschaft, der sozialen Verantwortung und dem Schutz des Lebens sowie der Lebensvoraussetzungen zugetan. Dahinter steht eine gemeinsame Verpflichtung: Wahrung und Entfaltung der Kultur in einem vielfältig geprägten Gemeinwesen.

\section{Föderalismus und Regionalpolitik}

Über diesen differenziert agierenden, föderalistisch konzipierten Staat legt sich nun eine Regionalpolitik, welche sich strukturschwacher Gebiete annimmt, im besonderen das Berggebiet fördert, periphere Räume stützt, zentralörtliche Lasten der Städte mindert und hinter allem die Aufgabe sieht, den Finanzausgleich so zu konzipieren, daß die problembeladenen Teilräume (Agglomerationen, Grenzgebiete usw.) ihre Hausaufgaben erfüllen können. Raumordnungs- und umweltbezogene Aufgaben werden zusätzlich damit verbunden.

«Föderalismus» und "Regionalpolitik» müssen auseinandergehalten werden. Beide sind vieldeutig. Dennoch, dies sei vorweggenommen, gibt es eine Gemeinsamkeit: Sowohl die Regionalpolitik als auch der Föderalismus befassen sich mit Teilräumen, die als bedeutsam für das Ganze anerkannt und gefördert werden. Trotzdem unterscheiden sie sich prinzipiell: Während der Begriffdes Föderalismus eng mit demjenigen des Bundesstaates verbunden ist, gilt dies nicht für die Regionalpolitik. Sogar Zentralstaaten - wie Frankreich - oder internationale Gemeinschaften ohne Staatsverfassung - so die EU und in deren Rahmen die EG (Art. 130 c ff. sowie Art. 198 a ff. EGV) - kennen eine breit angelegte Regionalpolitik. Sie ist also nicht für föderalistische Staaten typisch, sondern findet sich überall, wo teilräumliche Funktionen beachtet werden. Immer geht es darum, den Regionen einen gewissen Eigenwert zuzuerkennen, das ihnen eigene Potential zu entwickeln, ihr wirtschaftliches Wohlergehen zu fördern und interregionale Disparitäten abzubauen. Insofern wohnt jeder Art von Regionalpolitik - selbst in Zentralstaaten - ein gewisses "föderatives» Element inne. Auf der andern Seite sehen die Bundesstaaten im föderativen Element vorweg die politische Aufgabe, die staatliche Macht zu dezentralisieren, bürgernahe $\mathrm{Ge}$ bietskörperschaften - Gliedstaaten - öffentliche Aufgaben versehen zu lassen und sie an der gesamtstaatlichen Willensbildung zu beteiligen. Vereinfacht kann man sagen, das föderative Element sei staatsrechtlich und staatspolitisch angelegt, das regionalpolitische sei eher wirtschaftlich und lebensraumbezogen ausgerichtet.

\section{Das Subsidiaritätsprinzip}

Föderalismus und Regionalpolitik werden durch einen dritten Begriff zusätzlich belastet: Subsidiarität. Vorweg in der EU ist davon die Rede (Art. 3 b EGV). Der Begriff wurde hier wohl deshalb gewählt, weil sich das Wort «Föderalismus» nicht ohne weiteres ins Englische übersetzen läßt. In dieser Sprache bedeutet es nämlich genau das Gegenteil der deutschsprachigen Sinngebung, indem es auf die zentrale Macht und nicht auf die Gliedstaaten verweist.

Martin Lendi, Prof. Dr., o. Prof. für Rechtswissenschaft, ETH Zentrum, 8092 Zürich 
Für die Schweiz schließt das Subsidiaritätsprinzip zwei zentrale Aussagen ein, nämlich: - erstens - der Staat soll nur jene Aufgaben an die Hand nehmen, welche die Wirtschaft nicht bewältigen kann, und - zweitens - der Bund soll die öffentlichen Aufgaben, soweit es verantwortbar ist, auf die Gliedstaaten mit dem Ziel der Problemlösung an Ort und Stelle übertragen oder bei ihnen belassen, unter Inkaufnahme einer weniger effizienten, dafür aber demokratisch legitimierten Aufgabenbewältigung. Der Vertrag von Maastricht vernachlässigt den ersten Gesichtspunkt, und unter dem zweiten sieht er das Subsidiaritätsprinzip in Nuancen anders, nämlich ausgerichtet auf Effizienzkriterien.

\section{Ziele der Regionalpolitik, Ziele des Föderalismus}

Für den Föderalismus sind die Ziele leichter zu bestimmen als für die Regionalpolitik. Da die föderativen staatsrechtlich und staatspolitisch geprägt sind, sind sie von dorther faßbar. Es geht - abwehrend - um Machtteilung innerhalb des Staates, um Gegengewichte gegenüber einer egalitären Mehrheitsdemokratie, sodann aber - positiv - um die kulturelle, politische und gesellschaftliche Differenzierung als kraftvolle Basis eines menschennahen politischen, wirtschaftlichen und soziokulturellen Lebens. Die Regionalpolitik kann zum Anliegen der soziokulturellen Vielfalt ja sagen; im übrigen aber sucht sie die regionale Wettbewerbsfähigkeit und den Ausgleich der räumlichen Entwicklungsunterschiede, stets unter Ausschöpfung und Stärkung der regionalen Potentiale. Ob der Akzent für die Regionalpolitik eher - zukunftsgerichtet - auf der Förderung der endogenen Kräfte oder ausgehend von dem, was war - auf den Unterschieden der Vergangenheit beruht, ist eine Frage des Funktionsverständnisses der Regionalpolitik. So oder so kommt aber der Regionalpolitik die föderative Zielsetzung entgegen. Dort, wo sie im Staatsverständnis fehlt, muß sich die Regionalpolitik mit Fragen der teilräumlichen Gliederung intensiver befassen als in Bundesstaaten. So kommt es nicht von ungefähr, daß innerhalb der EU und in Zentralstaaten die «Regionalisierungsdebatte» im Zusammenhang der Regionalpolitik einen höheren Stellenwert hat als in Bundesstaaten.

\section{Ausprägung der Regionalpolitik im föderativen Staat}

Gemäß der Schweizerischen Bundesverfassung kommt, wie wir zeigen konnten, dem Föderalismus eine hohe Bedeutung zu. Er ist Teil der Verfassung und gibt dem schweizerischen Staat Struktur (Art. 3, 89, 121 BV). Für die Regionalpolitik gilt dies nicht im gleichen Sinn. Sie muß deshalb auf die vorgegebene Staatsstruktur Rücksicht nehmen. Außerdem hat sie unter Beachtung der Wirtschaftsfreiheit (Art. $31 \mathrm{BV}$ ) damit umzugehen, daß die staatlichen Möglichkeiten der Einflußnahme auf die wirtschaftliche Entwicklung begrenzt sind. Auf der andern Seite kommt ihr die staatspolitische Zusage entge- gen, daß Aufgaben auch dann auf unterer Stufe in den Teilräumen zu erfüllen sind, wenn sie zwar weniger effizient angegangen werden, dafür aber an Ort und Stelle sachgerechte Lösungen ermöglichen, die demokratisch akzeptiert sind.

Mit Rücksicht auf die staatsrechtlichen Vorgaben bleibt in der Schweiz für einen (blinden) Regionalismus der Bildung neuer Regionen wenig bis kein Raum. Das Zusammenführen von einzelnen Gebietskörperschaften oder die zusätzliche territoriale Neuorganisation in Richtung einer "Gebietsreform» haben selten oder gar keine Chance. Die historisch gewordene Gebietseinteilung dominiert. Ebenso kann die Regionalpolitik aus eigenem Sachpotential heraus nur begrenzt wirken, da ihre Mittel, Instrumente und Maßnahmen limitiert sind. Nichtmarktwirtschaftliche Maßnahmen stehen ohnehin nur begrenzt zur Disposition (Art. $31^{\text {bis }} \mathrm{BV}$ ). Sie muß sich deshalb vorweg als "Querschnittsaufgabe» verstehen und ihre Strategien und Konzepte derart einbringen, daß die in regionalpolitisch relevanten Belangen sachkompetenten Staatsorgane des Bundes, der Kantone und der Gemeinden «regionalpolitisch sinnvoll» agieren und den inneren Kräften der Regionen Auftrieb verleihen. Raumordnungs-, Verkehrs-, Steuer- und Finanzausgleichs-, Arbeitsmarktpolitik usw. müssen sich zusammenfinden, um regionalpolitisch wirksam zu werden. Bund, Kantone und Gemeinden sind gehalten, sich dabei - durchgehend, vertikal und horizontal - die Hand zu reichen.

\section{Regionen - am Beispiel der föderativen Schweiz}

Die gebiets- und bevölkerungsmäßig relativ kleine Schweiz mit ihrer differenzierten föderalistischen Staatsstruktur tut sich, wie nicht anders zu erwarten, schwer mit einer zusätzlichen regionalen Ebene. Europaweit betrachtet ist sie ohnehin nicht mehr als eine Region. Die Schweiz zählt beispielsweise weniger Einwohner als das deutsche Bundesland Baden-Württemberg. Innerhalb der Kantone, die von unterschiedlicher Größe sind, läßt sich nur in einigen wenigen größeren Kantonen (Zürich, Bern, Waadt, Graubünden, Wallis) daran denken, Regionen auszuscheiden; aber auch hier würde eine vierte Staatsebene (Bund, Kantone, Regionen, Gemeinden) mehr Probleme schaffen als lösen.

Trotzdem wird die regionale Betrachtungsweise gepflegt und in Sonderfragen auch umgesetzt. Ein besonders attraktives Beispiel bildet der Verkehrsverbund Zürich, der vom Kanton in Zusammenarbeit mit den SBB und den berührten Gemeinden getragen wird. Umgekehrt ist für die Agglomeration Zürich, die in die Kantone Aargau, Zug, Schwyz, Thurgau und St.Gallen hineinreicht, bis heute keine Trägerschaft gefunden worden. Die Berggebietsregionen sind in der Regel kantonsintern eingebettet; als hoheitlich agierende Gebietskörperschaften sind sie aber nicht organisiert. Auf der andern Seite werden unendlich viele interkommunale Belange (Abwasser, Wasserversorgung, Pflegeheime, Altersheime usw.) und interkantonale Aufgaben (Fachhochschulen, Einrich- 
tungen des Gesundheitswesens, Energieproduktion usw.) als regionale durch öffentlich-oder privatrechtliche Zweckverbände erfüllt, die sich in der Regel auf zwischengemeindliche resp. interkantonale Vereinbarungen stützen.

Im Verlauf der Jahrzehnte hat es immer wieder Entwürfe für Regionsbildungen gegeben, sei es auf überkantonaler Stufe, sei es auf der Ebene zwischen Kanton und Gemeinden, doch sind sie alle an den durch die föderative Staatsverfassung politikwirksamen Kantonen und Gemeinden angestoßen. Deren Aufgaben- und Kompetenzfülle setzt den Regionen von vornherein Grenzen. Das markanteste Beispiel entwickelte eine Neueinteilung des Bundes in vier Regionen, unter Verzicht auf die 26 Kantone. Dies ist aber in einem traditionsreichen föderalistischen Land nicht realisierbar - und auch nicht nötig. Die Kantone und Gemeinden sind in der Lage, regionalpolitische Anliegen aufzugreifen und umzusetzen, nötigenfalls in Kooperation mit dem Bund.

\section{Föderalismus: \\ Gegenstand oder Voraussetzung der Regionalpolitik?}

Föderalismus und Regionalpolitik bilden nach dem Gesagten keinen sich ausschließenden Gegensatz. Es gilt aber zu beachten: Die Regionalpolitik nimmt in einem föderativ verfaßten Bundesstaat mit marktwirtschaftlicher Ausrichtung andere, begrenztere Aufgaben wahr als in einem Zentralstaat und/oder in einem Staat mit einer betont staatlich gelenkten Wirtschaftspolitik. Die abstrakte Frage nach dem Verhältnis von Föderalismus und Regionalpolitik kann in der Theorie gestellt und von den Zielen und der Funktion her auch mindestens annäherungsweise beantwortet werden. Sie verliert aber an Schärfe und Grundsätzlichkeit unter den konkreten $\mathrm{Ge}$ gebenheiten eines Staates. Im Bundesstaat mit einer verfaßten föderativen Ordnung wird die Regionalpolitik faktisch und praktisch - auf dieser aufbauen und sie mithin als gegebene Voraussetzung erfassen, während sie im Zentralstaat und im Staat der zentral gelenkten Wirtschaft die teilräumliche Gliederung (Regionalisierung) nicht nur organisatorisch - als konkreten Gegenstand ihrer Bemühungen sehen muß, im Bewußtsein, daß dieser Aspekt rasch staatspolitische Dimensionen annimmt, da das Problem der innerstaatlichen Gliederung den Staat in seinem Verständnis trifft.
Die lexikonhafte Definition der Regionalpolitik als Politik der Berücksichtigung unterschiedlicher Voraussetzungen und Möglichkeiten der einzelnen Teilgebiete zur Entfaltung wirtschaftlicher und politischer Aktivitäten mag allgemeingültig klingen; sie verschließt aber die Augen vor der politischen Brisanz des Ansprechens von Teilräumen. Selbstredend kann die Regionalpolitik mit unterschiedlich definierten Regionen diesseits der rechtlich strukturierten innerstaatlichen Gliederung agieren, doch mit dem Vorstoß in die Teilräume lassen sich politische Weiterungen nicht vermeiden. Über sie entscheidet letztlich nicht die Regional-, sondern die Staatspolitik.

\section{Staatspolitik und Regionalpolitik}

Weder ersetzt der Föderalismus die Regionalpolitik, noch entbindet die Regionalpolitik die Staatspolitik von der Auseinandersetzung mit den innerstaatlichen Teilräumen. Die Regionalpolitik tut deshalb gut daran, mit der Staatspolitik in engem Kontakt zu bleiben, wie umgekehrt die Staatspolitik davon lebt, daß die «Teilräume des Staates» kulturell, politisch, wirtschaftlich, gesellschaftlich und ökologisch so gesund und stark sind, daß der Staat insgesamt seine Kohärenz und Identität findet.

\section{Literaturhinweise}

HERZOG, R. (1987): Subsidiaritätsprinzip, Evangelisches Staatslexikon, 3. A., S. $3750 \mathrm{f}$.

LENDI, M. (1984): Schweizerische Regionalpolitik. In: Recht und Politik der Raumplanung, Zürich, S. 217 ff. (siehe auch ZBI 84/1983, S. 241 ff.).

LENDI, M. (1995): Le principe de subsidiarité au sein de la Fédération suisse. In: EUREC (Europäische Zeitschrift für Regionalentwicklung) 2/95, S. $22 \mathrm{ff}$.

OSSENBÜHL, F. (Hrsg.) (1990): Föderalismus und Regionalismus in Europa, Baden-Baden (daselbst EICHENBERGER, K., Länderbericht Schweiz, S. 30).

SCHINDLER, D. (1992): Schweizerischer und europäischer Föderalismus, ZBI 5/92, $193 \mathrm{ff}$.

SCHWEIZERISCHER BUNDESRAT (1996): Botschaft über die Neuorientierung der Regionalpolitik, Bern (im Erscheinen).

THÜRER, D. (1986): Bund und Gemeinden, Berlin/Heidelberg. 